Emergent matter from 3D generalized group field theories

This article has been downloaded from IOPscience. Please scroll down to see the full text article.

2010 Class. Quantum Grav. 27145006

(http://iopscience.iop.org/0264-9381/27/14/145006)

View the table of contents for this issue, or go to the journal homepage for more

Download details:

IP Address: 194.94.224.254

The article was downloaded on 02/04/2013 at 12:27

Please note that terms and conditions apply. 


\title{
Emergent matter from 3D generalized group field theories
}

\author{
Alessandro Di Mare and Daniele Oriti \\ Albert Einstein Institute, Am Muehlenberg 1, D-14476 Golm, Germany \\ E-mail: aldimare@aei.mpg.de and doriti@aei.mpg.de
}

Received 25 January 2010, in final form 12 April 2010

Published 28 May 2010

Online at stacks.iop.org/CQG/27/145006

\begin{abstract}
We identify classical solutions of a generalized group field theory (GFT) model in three dimensions and study the corresponding perturbations, deriving their effective dynamics. We discuss their interpretation as emergent matter fields. This allows us on the one hand to test the proposed mechanism for emergence of matter as a phase of GFT, and on the other hand to expose some limitations of the generalized GFT formalism.
\end{abstract}

PACS numbers: 04.60.- m, 04.60.Pp, 11.15.-q

\section{Introduction}

In recent years several approaches to quantum gravity have been developed, with important results [1]. Among them, group field theories (GFTs) [2-4] are, in our opinion, particularly promising. GFTs are quantum field theories defined over a group manifold, representing a 'meta-space' of discrete spacetime geometries and not spacetime itself, as a new algebraic and combinatorial realization of the 'third quantization' idea [5]. Moreover, GFTs bring together most of the ingredients entering in other non-perturbative and background-independent approaches (such as loop quantum gravity, spin foam models and simplicial quantum gravity approaches) [2, 3]. Very little is known, still, about GFT models for quantum gravity, in both three and four dimensions, and a lot of technical work should go into their analysis, for example their classical solutions, which will be one focus of the present work, and their purely field theoretic aspects, which are the subject of attention at present [6-9].

An important issue in many (non-perturbative) quantum gravity formalisms is the inclusion and the correct description of matter fields. In the literature, we can distinguish two different strategies. One approach is to start from a model describing a quantum spacetime without matter and then add degrees of freedom describing matter, such as fields, point particles or extended objects. This is the standard route both in loop quantum gravity [10,11], spin foam models [12-16] and simplicial quantum gravity [17, 18]. In a GFT context this means, for example, writing down a coupled GFT action [19] for both gravity and matter fields that would produce, in perturbative expansion, a sum over simplicial complexes with dynamical geometry 
together with Feynman graphs for the matter fields living on the simplicial complexes. Or it means [20] defining a GFT model whose Feynman amplitudes can be understood as state sum models for gravity with appropriate matter field observables. An alternative approach is to think of matter degrees of freedom as a subset of the same degrees of freedom defining the microstructure of quantum spacetime itself and emerge as fluctuations around a background configuration of the same. This idea, pursued for example (in different ways) in [21-24], shares some similarities with analog gravity models in condensed matter systems, in which quasi-particles with an effective description as matter fields propagating on curved geometries emerge as fluctuations around stable vacua of the underlying many-body system (e.g. a Bose condensate), but are of course collective excitations of the same microscopic degrees of freedom just as well. A strategy for emergent matter within the GFT framework, in particular, first studied in [23-25], is based on the following idea: starting from a fundamental GFT action, one looks for solutions of the corresponding equation of motion; then, one considers perturbations around these solutions, obtaining an effective dynamics for the perturbation field. The task is to identify a class of solutions and of perturbations, such that the effective dynamics for these perturbations takes the form of a matter field theory on an effective spacetime.

It turns out [25] that the effective matter field theories emerging from GFTs following this procedure are non-commutative quantum field theories on non-commutative spacetimes with a Lie algebra structure, and with a curved momentum (group) manifold. This only makes this strategy all the more interesting. In fact, non-commutative geometry has been often advocated as the appropriate language to describe a quantum spacetime, i.e. a regime which, already far from the full non-perturbative quantum gravity dynamics at the (ultra)Planckian scales, is still semi-classical in that it incorporates somehow the quantum gravity corrections to continuum physics at the effective level. This can take the form of an effective minimal (Planck) length scale in matter field theories of a non-trivial commutation relation between position operators in quantum mechanics (so that the notion of spacetime point, and of spacetime continuum, becomes meaningless) or of a generalized uncertainty principle, or of a form of co-gravity, i.e. curvature in momentum space [26, 27]. It is actually this sort of effect, a curved momentum sector for particle (and field) kinematics, which shows up most naturally in a GFT context. Moreover, in recent years, much work in the context of quantum gravity phenomenology [28] was developed. On the one hand, this starts from the simple idea that several astrophysical systems could work as a magnifying lens to amplify quantum gravity effects even if Planck scale suppressed, and make them accessible to experiments. On the other hand, much of this work relies on effective models of quantum gravity, rather than on the tentative fundamental formalisms currently available, and in particular on effective non-commutative models for matter kinematics and dynamics, one important example being the so-called deformed (or doubly) special relativity [29]. This means that the recent results on the emergence of effective non-commutative matter field theories from GFT have the potential to help bridging the gap between the microscopic dynamics they define for quantum space and macroscopic physics and quantum gravity phenomenology.

In this paper we thus follow this strategy and apply it to a generalized class of GFT models introduced in [30-32], focusing on the 3D case. This generalized GFT formalism has been introduced as an attempt to formulate GFT models, which on the one hand depended explicitly on metric-related variables, thus allowing a more manifest encoding of simplicial geometry at the level of the GFT action, and on the other hand possessed Feynman amplitudes with the explicit form of a simplicial gravity path integral, thus clarifying the relation between spin foam and simplicial gravity structures. Obviously, the viability and correctness of such a generalized GFT formalism is something to be tested. Therefore, we understand the results of this paper in two dual ways: (1) as a test of the strategy for the emergence matter from GFTs 
as perturbations around classical GFT solutions; in fact, we check here whether this strategy works nicely also for (much) less trivial models than those it has been applied to so far, and under which assumptions and conditions; (2) as a test of the generalized GFT formalism itself; in fact, we will see that some difficulties encountered in applying this procedure, and some unsatisfactory features of the resulting effective actions for perturbations, can naturally be understood as stemming from limitations of the generalized GFT formalism itself, rather than as failures of the 'emergent matter strategy'. Let us also stress that the same identification of classical solutions of the model and of the corresponding perturbative dynamics will be an interesting and highly non-trivial result, from a purely technical perspective.

The outline of the paper is as follows. In the remaining sections of the introduction, we recall how a non-commutative matter field theory for a scalar field emerges from the perturbations around classical GFT solutions in a simple 3D model, and then we introduce the 3D generalized GFT model. In section 2, we consider a simplified version of the latter, identify a class of solutions of the classical GFT equations, and then study the corresponding perturbations; we will see that the basic elements of the complete analysis of perturbations to be done on the full model are already evident, together with the limitations of the same model in this respect. In section 3, we move on to the complete model; we find some exact and approximate classical solutions of its equations of motion, and extract the effective dynamics for the corresponding perturbations, focusing on the approximate case. We conclude with a discussion of the results obtained and an outlook on further developments, as suggested also by our results.

\subsection{Non-commutative matter from GFT}

In this section we briefly review some results about the emergence of an effective noncommutative field theory in the 3D case [23]. We start from the following GFT action (Boulatov model), based on a group manifold being either $S O(3)$ or $S U(2)$ :

$$
\begin{aligned}
S[\phi]=\frac{1}{2} \int \mathrm{d} g_{1} \mathrm{~d} g_{2} \mathrm{~d} g_{3} \phi\left(g_{1}, g_{2}, g_{3}\right) \phi\left(g_{3}, g_{2}, g_{1}\right) \\
\quad-\frac{\lambda}{4 !} \int \mathrm{d} g_{1} \ldots \mathrm{d} g_{6} \phi\left(g_{1}, g_{2}, g_{3}\right) \phi\left(g_{3}, g_{4}, g_{5}\right) \phi\left(g_{5}, g_{2}, g_{6}\right) \phi\left(g_{6}, g_{4}, g_{1}\right),
\end{aligned}
$$

where $\phi$ is a real field that we suppose invariant under diagonal action of the group $\phi\left(g_{1}, g_{2}, g_{3}\right)=\phi\left(g_{1} h, g_{2} h, g_{3} h\right)$. The corresponding classical equation of motion is

$\phi\left(g_{3}, g_{2}, g_{1}\right)=\frac{\lambda}{3 !} \int \mathrm{d} g_{4} \mathrm{~d} g_{5} \mathrm{~d} g_{6} \phi\left(g_{3}, g_{4}, g_{5}\right) \phi\left(g_{5}, g_{2}, g_{6}\right) \phi\left(g_{6}, g_{4}, g_{1}\right)$

and a class of solutions of the same equation is parametrized by a function $f: G \rightarrow \mathbb{R}$, satisfying $\int_{G} \mathrm{~d} g f^{2}(g)=1$ and given by

$$
\phi_{f}\left(g_{1}, g_{2}, g_{3}\right)=\sqrt{\frac{3 !}{\lambda}} \int_{G} \mathrm{~d} h \delta\left(g_{1} h\right) f\left(g_{2} h\right) \delta\left(g_{3} h\right) .
$$

Since the Boulatov model defines a (third) quantization of BF theory, whose only configurations are locally flat geometries on arbitrary topology, such solutions can be interpreted as describing a quantum flat space. Now we want to study classical perturbations around these solutions, in particular those of the form $\psi\left(g_{1}, g_{2}, g_{3}\right) \equiv \psi\left(g_{1}, g_{3}\right)=\psi\left(g_{1} g_{3}^{-1}\right)$. 
For them we obtain the following effective action:

$$
\begin{aligned}
S^{(f)}[\psi] \equiv S\left[\phi_{f}+\psi\right]-S\left[\phi_{f}\right]=\int \mathrm{d} g \psi(g) \mathcal{K}_{f}(g) \psi\left(g^{-1}\right) \\
-\frac{\mu}{3 !} \int \mathrm{d} g_{1} \mathrm{~d} g_{2} \mathrm{~d} g_{3} \delta\left(g_{1} g_{2} g_{3}\right) \psi\left(g_{1}\right) \psi\left(g_{2}\right) \psi\left(g_{3}\right) \\
-\frac{\lambda}{4 !} \int \mathrm{d} g_{1} \mathrm{~d} g_{2} \mathrm{~d} g_{3} \mathrm{~d} g_{4} \delta\left(g_{1} \ldots g_{4}\right) \psi\left(g_{1}\right) \psi\left(g_{2}\right) \psi\left(g_{3}\right) \psi\left(g_{4}\right),
\end{aligned}
$$

with the kinetic term given by

$$
\mathcal{K}_{f}(g)=\frac{1}{2}\left[1-2\left(\int \mathrm{d} h f(h)\right)^{2}-\int \mathrm{d} h f(h) f(h g)\right] .
$$

The nature of this action as an effective scalar field theory on a non-commutative space is evident once we interpret the group manifold on which it is defined as momentum space, so that the constraints $\delta\left(g_{1} g_{2} g_{3}\right)$ and $\delta\left(g_{1} \ldots g_{4}\right)$ represent the momentum conservation. As a curved space, this momentum space is dual to a non-commutative configuration space with a Lie algebra structure (given by the $\mathfrak{s u}(2)$ ). This duality can be made explicit by introducing a Fourier transform mapping functions over the group to functions over the corresponding algebra [33, 34], using which we can rewrite the above action in terms of functions on $\mathbb{R}^{3}$ endowed with a non-commutative $\star$-product $[33,34]$, which can then be shown to be invariant under the quantum double of $S U$ (2), a deformation of the Euclidean 3D Poincaré group.

Similar results have been obtained also in the 4D case and in Lorentzian signature [24], where it has been shown that, starting from a GFT model for the $S O(4,1)$ BF theory, one can obtain an effective non-commutative field theory of the DSR type, i.e. for a scalar field living on the $\kappa$-Minkowski spacetime and with a de Sitter space of momenta. Obviously, this is a model of a more direct interest for quantum gravity phenomenology.

We can conclude that perturbations around a non-trivial quantum geometric background, defined by a solution of the GFT equations, follow the dynamics of an effective scalar field theory of a non-commutative type. So we can interpret matter as a phase of quantum geometry, therefore already contained in our quantum gravity models. Moreover, this type of analysis of GFT perturbations is useful to expose the deep link between the GFT formalism and non-commutative geometry.

\subsection{Generalized GFT model}

A generalized GFT formalism has been introduced in [30] and further developed in both 3D and $4 \mathrm{D}$ in $[31,32]$, its purpose being encoding and controlling in a more explicit way simplicial geometry at the level of the GFT action, and obtaining Feynman amplitude with the manifest form of a simplicial gravity path integral. In turn, this should serve as a complete definition of the quantum dynamics of simplicial structures, to obtain a spin foam model with a clear underlying geometry and to strengthen the links between spin foam and simplicial gravity approaches.

In the $3 \mathrm{D}$ case, the fundamental variable is a real function of three group variables and three algebra variables: $\phi: G^{3} \times \mathfrak{g}^{3} \rightarrow \mathbb{R}$, which satisfies $\phi\left(g_{1}, g_{2}, g_{3} ; B_{1}, \ldots, B_{3}\right)=$ $\phi\left(g_{1} h, g_{2} h, g_{3} h ; h B_{1} h^{-1}, h B_{2} h^{-1}, h B_{3} h^{-1}\right)$. The group and Lie algebra variables are interpreted as the discrete triad and connection variables of simplicial BF theory or, equivalently, of discrete 3D gravity. Its classical action is as follows: 


$$
\begin{aligned}
& S=\frac{1}{2} \frac{1}{(2 \pi)^{9}} \int_{G^{3}} \mathrm{~d} g_{1} \mathrm{~d} g_{2} \mathrm{~d} g_{3} \int_{\mathfrak{g}^{3}} \mathrm{~d} \vec{B}_{1} \mathrm{~d} \vec{B}_{2} \mathrm{~d} \vec{B}_{3} \phi_{123} \hat{O}_{1} \hat{O}_{2} \hat{O}_{3} \phi_{123}^{*} \\
&-\frac{\lambda}{4 !(2 \pi)^{36}} \int_{G^{6}} \mathrm{~d} g_{1} \ldots \mathrm{d} g_{6} \int_{\mathfrak{g}^{6}} \mathrm{~d} \vec{B}_{1} \ldots \mathrm{d} \vec{B}_{6} \phi_{123} \phi_{345} \phi_{526} \phi_{641}
\end{aligned}
$$

where we have introduced the operator $\hat{O}=\square_{G}+B^{2}+\frac{m^{2}}{8}$, with $\square_{G}$ being the Laplace-Beltrami operator on the group manifold $G$, and the notation $\phi_{123}=\phi\left(g_{1}, g_{2}, g_{3} ; B_{1}, B_{2}, B_{3}\right)$.

Note that even though the field is a function of Lie algebra triad variables, it is treated in this formalism as an ordinary function, and the Lie algebra itself is considered in its vector space aspects only, so that integrations are performed by means of the Lebesgue measure and no star product is introduced when multiplying more than one GFT field. This will have important consequences when studying the corresponding perturbations around classical solutions.

For the achievements and limitations of this formalism concerning its original goals mentioned above, we refer to the literature. Here we will only try to see whether the results on perturbations obtained for the Boulatov model extend to this more complicated case, and at the same time use this analysis of perturbations as a test for the formalism, trying to expose further its limitations by making them explicit at the level of the resulting effective dynamics.

\section{The static-ultralocal case}

Let us begin from the analysis of the simpler 'static-ultralocal' truncation of the model. It corresponds to the case in which the kinetic operator is 'freezed' to the identity, imposing $\hat{O}=\mathbb{I}$ in (6):

$$
\begin{aligned}
& S=\frac{1}{2} \frac{1}{(2 \pi)^{9}} \int_{G^{3}} \mathrm{~d} g_{1} \mathrm{~d} g_{2} \mathrm{~d} g_{3} \int_{\mathfrak{g}^{3}} \mathrm{~d} \vec{B}_{1} \mathrm{~d} \vec{B}_{2} \mathrm{~d} \vec{B}_{3} \phi_{123} \phi_{321} \\
& \quad-\frac{\lambda}{4 !(2 \pi)^{36}} \int_{G^{6}} \mathrm{~d} g_{1} \ldots \mathrm{d} g_{6} \int_{\mathfrak{g}^{6}} \mathrm{~d} \vec{B}_{1} \ldots \mathrm{d} \vec{B}_{6} \phi_{123} \phi_{345} \phi_{526} \phi_{641} .
\end{aligned}
$$

In the following we always assume that solutions depend on the moduli of the vectors $B$, and not from its direction, so that the invariance property of the field under the diagonal group action on the Lie algebra arguments $h B h^{-1}$ is trivialized. Moreover, to simplify the notation, we define $B=|\vec{B}|$. Anyway we use the notation $\int \mathrm{d} \vec{B}$ to distinguish integration over all algebra with integration over only the module $\int \mathrm{d} B$.

\subsection{Solutions of the equations of motion}

The equation of motion corresponding to the above action is

$$
\phi_{123}=\frac{\lambda}{3 !} \frac{1}{(2 \pi)^{27}} \int_{G^{3}} \mathrm{~d} g_{4} \mathrm{~d} g_{5} \mathrm{~d} g_{6} \int_{\mathfrak{g}^{3}} \mathrm{~d} \vec{B}_{4} \mathrm{~d} \vec{B}_{5} \mathrm{~d} \vec{B}_{6} \phi_{345} \phi_{526} \phi_{641}
$$

and it is easy to verify that the following class of functions, a simple generalization of (3), define classical solutions:

$$
\varphi_{123}=\sqrt{\frac{3 !(2 \pi)^{27}}{\lambda}} \int_{G} \mathrm{~d} h \delta\left(g_{1} h\right) \psi_{1}\left(B_{1}\right) f\left(g_{2} h ; B_{2}\right) \psi_{3}\left(B_{3}\right) \delta\left(g_{3} h\right),
$$

where the functions $f, \psi_{1}$ and $\psi_{3}$ have to fulfill the following relations:

$$
\int_{G} \mathrm{~d} g \int_{\mathfrak{g}} \mathrm{d} \vec{B} f^{2}(g, B)=1 \int_{\mathfrak{g}} \mathrm{d} \vec{B} \psi_{1}(B) \psi_{3}(B)=1 .
$$

We also require, for simplicity, the function $f(g, B)$ to be separable in its variables: $f(g, B)=f_{1}(g) f_{2}(B)$. 


\subsection{Perturbations}

Let us consider a generic perturbation $\epsilon\left(g_{1}, g_{2}, g_{3} ; B_{1}, B_{2}, B_{3}\right)$ and compute the effective action

$$
\begin{aligned}
S[\epsilon] \equiv & S[\varphi+\epsilon]-S[\varphi] \\
= & \frac{1}{2} \int[\mathrm{d} g] \int[\mathrm{d} \vec{B}] \epsilon_{123} \epsilon_{321}-\int[\mathrm{d} g] \mathrm{d} h_{1} \int[\mathrm{d} \vec{B}] \psi_{1}\left(B_{5}\right) f_{1}\left(g_{2} h_{1}\right) f_{2}\left(B_{2}\right) f_{1}\left(g_{4} h_{1}\right) \\
& \times f_{2}\left(B_{4}\right) \psi_{3}\left(B_{1}\right) \epsilon\left(h_{1}^{-1}, g_{2}, g_{3} ; B_{1}, B_{2}, B_{3}\right) \epsilon\left(g_{3}, g_{4}, h_{1}^{-1} ; B_{3}, B_{4}, B_{5}\right) \\
& -\frac{1}{2} \int[\mathrm{d} g] \mathrm{d} h_{1} \mathrm{~d} h_{2} \int[\mathrm{d} \vec{B}] \psi_{1}\left(B_{3}\right) f_{1}\left(g_{4} h_{1}\right) f_{2}\left(B_{4}\right) \psi_{3}\left(B_{1}\right) \psi_{3}\left(B_{5}\right) \psi_{1}\left(B_{6}\right) \\
& \times f_{1}\left(g_{4} h_{2}\right) f_{2}\left(B_{4}\right) \psi_{3}\left(B_{6}\right) \epsilon\left(h_{2}^{-1}, g_{2}, h_{1}^{-1} ; B_{1}, B_{2}, B_{3}\right) \epsilon\left(h_{1}^{-1}, g_{2}, h_{2}^{-1} ; B_{5}, B_{2}, B_{6}\right) \\
& -\frac{\lambda}{4 !(2 \pi)^{27}} \int[\mathrm{d} g] \int[\mathrm{d} \vec{B}] \epsilon_{123} \epsilon_{345} \epsilon_{526} \epsilon_{641}-\sqrt{\frac{\lambda}{3 !(2 \pi)^{27}}} \int[\mathrm{d} g] \mathrm{d} h \int[\mathrm{d} \vec{B}] \psi_{1}\left(B_{6}\right) \\
& \times f_{1}\left(g_{4} h\right) f_{2}\left(B_{4}\right) \psi_{3}\left(B_{1}\right) \epsilon\left(h^{-1}, g_{2}, g_{3} ; B_{1}, B_{2}, B_{3}\right) \epsilon_{345} \epsilon\left(g_{5}, g_{2}, h^{-1} ; B_{5}, B_{2}, B_{6}\right),
\end{aligned}
$$

where we used the notation $\epsilon_{123}=\epsilon\left(g_{1}, g_{2}, g_{3} ; B_{1}, B_{2}, B_{3}\right)$, where convenient.

This is the action for a generic perturbation, only assumed to have the same diagonal invariance of the fundamental GFT field, but it simplifies considerably and can then be interpreted physically, by appropriate simplified choices on the perturbation field. In particular, having in mind the possible interpretation of the $B$ and $g$ variables as configuration and momentum variables, respectively, we try to reduce the dependence of the perturbation field to either of the two sectors.

Case 1: dynamical group sector. Now we show how one can recover the same effective action in [23] with a particular choice of the perturbation field. We assume a separable dependence on $g$ and $B$ variables (this is suggested also by the fact that neither the kinetic term nor the interaction term couples the two sectors), and the dependence on a single group variable via the combination $g_{1} g_{3}^{-1}: \epsilon\left(g_{1}, g_{2}, g_{3} ; B_{1}, B_{2}, B_{3}\right)=\phi\left(g_{1} g_{3}^{-1}\right) \xi\left(B_{1}, B_{2}, B_{3}\right)$. Factoring out the integration over the $B$ variables, we get the following effective action for the perturbation field $\phi(g)$ :

$$
\begin{gathered}
S[\phi]=\int \mathrm{d} g \phi(g) \mathcal{K}(g) \phi\left(g^{-1}\right)-\frac{\eta}{3 !} \int[\mathrm{d} g]^{3} \delta\left(g_{1} \ldots g_{3}\right) \psi\left(g_{1}\right) \ldots \psi\left(g_{3}\right) \\
-\frac{\mu}{4 !} \int[\mathrm{d} g] \delta\left(g_{1} \ldots g_{4}\right) \psi\left(g_{1}\right) \ldots \psi\left(g_{4}\right) .
\end{gathered}
$$

Here, the kinetic term is given by

$$
\mathcal{K}_{f}(g)=\frac{1}{2}\left[\alpha-2 \beta\left(\int \mathrm{d} h f(h)\right)^{2}-\gamma \int \mathrm{d} h f(h) f(h g)\right],
$$

which is the same as that of action (4), with up to some appropriate redefinition of coupling constants:

$\alpha=\int \mathrm{d} \vec{B}_{1} \mathrm{~d} \vec{B}_{2} \mathrm{~d} \vec{B}_{3} \xi\left(B_{1}, B_{2}, B_{3}\right) \xi\left(B_{3}, B_{2}, B_{1}\right)$,

$\beta=\int \mathrm{d} \vec{B}_{1} \ldots \mathrm{d} \vec{B}_{5} \psi_{1}\left(B_{5}\right) f_{2}\left(B_{2}\right) f_{2}\left(B_{4}\right) \xi\left(B_{1}, B_{2}, B_{3}\right) \xi\left(B_{3}, B_{4}, B_{5}\right)$,

$\gamma=\int \mathrm{d} \vec{B}_{1} \ldots \mathrm{d} \vec{B}_{6} \psi_{1}\left(B_{3}\right) f_{2}\left(B_{4}\right) \psi_{3}\left(B_{5}\right) \psi_{1}\left(B_{6}\right) f_{2}\left(B_{4}\right) \psi_{3}\left(B_{6}\right) \xi\left(B_{1}, B_{2}, B_{3}\right) \xi\left(B_{3}, B_{4}, B_{5}\right)$, 


$$
\begin{aligned}
\eta= & \sqrt{6 \lambda} \int \mathrm{d} g f_{1}(g) \int \mathrm{d} \vec{B}_{1} \ldots \mathrm{d} \vec{B}_{6} \psi_{1}\left(B_{6}\right) \psi_{3}\left(B_{1}\right) f_{2}\left(B_{4}\right) \xi\left(B_{1}, B_{2}, B_{3}\right) \\
& \quad \times \xi\left(B_{3}, B_{4}, B_{5}\right) \xi\left(B_{5}, B_{2}, B_{6}\right)
\end{aligned}
$$

Of course the perturbation field must be chosen such that this new set of coupling constants does not diverge, with the functional dependence on the $B$ sector to be considered as fixed, with no variation allowed in the classical theory and no fluctuations (functional integration) in the quantum theory. We see that we have recovered, even from the more complicated GFT formalism, the expected effective action for the perturbations that can be interpreted as a non-commutative scalar field theory written in momentum space. Even in this drastic simplification of the formalism, we also see, however, that the way we could obtain it was to completely decouple the $B$ and $g$ sector and to leave only this last one as dynamical.

Case 2: dynamical Lie algebra sector. Now we focus instead on the $B$ sector, and treat the group sector as non-dynamical. The idea is then to reproduce the same action as above, or a modification thereof, but written now in configuration space, according to the standard interpretation of the effective theory and of the variables appearing in the GFT outlined above.

As a regularization, we introduce a cut-off $B_{\max }$ in the $B$ integration, and the constant $V_{B}=4 \pi \int_{0}^{B_{\max }} \mathrm{d} B B^{2}$, that is the (truncated) volume of $\mathbb{R}^{3}$. We then consider a perturbation of the form

$$
\epsilon_{123}=\frac{1}{\sqrt{V_{B}}} \delta\left(\vec{B}_{1}-\vec{B}_{2}\right) \psi\left(\vec{B}_{2}+\vec{B}_{3}\right)
$$

with no dependence on group variables (note that this does not lead to any divergence because of the compact nature of the group manifold). The dependence on the three variables $B_{i}$ has been chosen to be what would have followed from a Fourier transform from $g$ to $B$ variables of the function we used as perturbation in the previous case. Moreover we introduce a new condition on the solution imposing that $\int \mathrm{d} g f(g)=0$. With this condition the integration over the group variables kills the cubic interaction and some quadratic terms. The resulting effective action for this perturbation field is

$$
S[\psi]=\frac{1}{2} \int \mathrm{d} \vec{B} \psi^{2}(B)+\frac{\lambda}{4 !} \int \mathrm{d} \vec{B} \psi^{4}(B),
$$

that is, an ordinary local $\lambda \phi^{4}$ theory with a trivial kinetic term (i.e. again a static-ultralocal field theory).

We can interpret this as a scalar field theory written in configuration space (given by $\mathbb{R}^{3}$ ). The triviality of the kinetic term arises directly from the initial ultra-locality restriction, but could possibly be lifted by some more involved choice of classical solution or perturbation field, at the cost of introducing derivatives in the same. The point to note, however, is that we find no sign of the expected underlying non-commutativity in this field theory. That is, while we find the expected local dependence on $B$ variables that matches their interpretation as configuration variables in some sense dual to the group variables, and while we could try to modify our construction to obtain a more complicated (and interesting) kinetic term or interaction, it is evident that the conjugate nature of $B$ and $g$ sectors is lost and cannot be recovered. Due to the simplicity of the underlying model, it is also clear that this conjugate nature fails to be present in the effective action because it fails to be correctly implemented in the original generalized GFT formalism. We will come back to this point in the following. 


\section{Full generalized model}

In this section we consider the complete action (6) and the corresponding integro-differential equation of motion:

$$
\hat{O}_{1} \hat{O}_{2} \hat{O}_{3} \phi_{321}=\frac{\lambda}{3 !(2 \pi)^{27}} \int_{G^{3}} \mathrm{~d} g_{4} \mathrm{~d} g_{5} \mathrm{~d} g_{6} \int_{\mathfrak{g}^{3}} \mathrm{~d} \vec{B}_{4} \mathrm{~d} \vec{B}_{5} \mathrm{~d} \vec{B}_{6} \phi_{345} \phi_{526} \phi_{641}
$$

\subsection{Exact solution of the equation and perturbations}

We first try to find an exact solution of this equation and to obtain an effective field theory for the perturbations. As it is apparent, the integro-differential equation (15) presents a very complicated structure and there is no standard or general procedure to solve it. For simplicity we look for solutions that are separable in the three sets of variables, i.e. of the type

$$
\phi_{123}=\int \mathrm{d} h \psi_{1}\left(g_{1} h, B_{1}\right) f\left(g_{2} h, B_{2}\right) \psi\left(g_{3} h, B_{3}\right),
$$

where we have imposed the usual invariance under diagonal right action of the group, by explicit projection. It should be clear that this is a choice and that the equation could have solutions which do not fulfill the requirement of separability ${ }^{1}$, and that more general type of functions can be considered. Unfortunately, we have not been able to identify any solution outside this simpler class of functions, despite intense efforts. Therefore, while we cannot exclude the existence of more general solutions, we are forced to work within this simpler class.

Now it is easy to show that any function $\phi_{123}$ of the above form is an exact solution of the GFT equation of motion if the following conditions are satisfied:

(1) the functions $\psi_{1}, f, \psi_{3}$ are solutions of the equation

$$
\left(\square+B^{2}\right) \phi(g, B)=0,
$$

so they are eigenfunctions of the operator $\hat{O}$ with the eigenvalue $-\frac{m^{2}}{8}$.

(2) $\psi_{1}, \psi_{3}$ and $f$ satisfy

$$
\int \mathrm{d} \vec{B} \int \mathrm{d} g \psi_{3}\left(g h_{1}, B\right) \psi_{1}\left(g h_{2}, B\right)=\delta\left(h_{1}^{-1} h_{2}\right) \quad \int \mathrm{d} \vec{B} \int \mathrm{d} g f^{2}(g, B)=1 .
$$

We now look for such functions, starting from condition (1). We now prove the following.

Proposition 1. Let $\mathcal{I}$ be the set of functions defined on the Cartesian product $G \times \mathfrak{g}$ and square integrable with respect to the group variable (so that we can apply the Peter Weyl theorem to decompose them in irreducible representations $j$ of $G$ ). The most general solution, in the set $\mathcal{I}$, of the equation (17) is of the form

$\phi(g, B)=\sum_{j, n, m} \tilde{\phi}_{j}^{n m}(B) D_{n m}^{j}(g) \quad$ with $\begin{cases}\phi_{m n}^{j}(B) & \text { arbitrary } \forall B: B^{2}=j(j+1) \\ \phi_{m n}^{j}(B)=0 & \text { elsewhere. }\end{cases}$

Proof. The proof is straightforward. Because of the Peter-Weyl theorem, every solution of the equation, belonging to the set $\mathcal{I}$, can be written as $\phi(g, B)=\sum_{j, n, m} \tilde{\phi}_{j}^{n m}(B) D_{n m}^{j}(g)$, for some coefficient functions $\phi_{m n}^{j}(B)$. Being a solution, it has to satisfy: $\sum_{j=0}^{\infty} \sum_{n, m}\{[-j(j+$

1 Note also that the requirement of a right group invariance couples the arguments of the functions, away from separability. 
$\left.\left.1)+B^{2}\right] \tilde{\phi}_{j}^{n m}(B)\right\} D_{n m}^{j}(g)=0$, which implies, since the $D_{m n}^{j}(g)$ is a complete orthonormal basis in $\mathcal{I}$, that $\left[-j(j+1)+B^{2}\right] \tilde{\phi}_{j}^{n m}(B)=0 \quad \forall j, n, m$. This equation can be satisfied only if the coefficient functions $\phi_{m n}^{j}(B)$ are null for all values such that $B^{2} \neq j(j+1)$, while they can be arbitrary otherwise.

Therefore, the functions (19), considered as a function of $\vec{B}$ are non-null only on spheres of radius $\sqrt{j(j+1)}$. Thus the integral over the algebra of these eigenfunctions is obviously zero, because each sphere is a set of null measure. Therefore, conditions (2) and (3) cannot be satisfied unless the functions $\tilde{\phi}_{j}^{n m}(B)$ are actually distributions on $\mathbb{R}^{3}$. The idea is then to define a three-dimensional delta-like function, i.e. null everywhere, except on the origin and on the spheres of radius $\sqrt{j(j+1)}$, with $j \in \mathbb{N}^{+}$, where it goes to infinity, and multiply it by a regular function of $B$ to define completely the functions $\tilde{\phi}_{j}^{n m}(B)$. There are many possibilities to define this distribution with a limit procedure, and we do it in the appendix, providing explicit examples. In the following we call that distribution $\alpha(\vec{B})$. Its only property we need is

$$
\int \mathrm{d} \vec{B} \alpha^{2}(\vec{B}) f(B)=\sum_{j=0}^{\infty}(2 j+1)^{2} f(\sqrt{j(j+1))}),
$$

where both integral and sum have to converge. Let $\psi_{1}(g, B)$ and $\psi_{3}(g, B)$ be solutions of (17). We also introduce the following distributions:

$$
\varphi_{1}(g, B)=\alpha(\vec{B}) \psi_{1}(g, B) \quad \varphi_{3}(g, B)=\alpha(\vec{B}) \psi_{3}(g, B) .
$$

These define solutions too, as they satisfy condition (1), as it can be easily checked. Now we consider condition (2) and compute the following integral:

$$
\begin{aligned}
\int \mathrm{d} g \int \mathrm{d} \vec{B} \varphi_{1}\left(g h_{1}, B\right) \varphi_{3}\left(g h_{2}, B\right) \\
\quad=\int \mathrm{d} g \int \mathrm{d} B \alpha^{2}(\vec{B}) \psi_{1}\left(g h_{1}, B\right) \psi_{3}\left(g h_{2}, B\right) \\
\quad=\sum_{j=0}^{\infty}(2 j+1)^{2} \int \mathrm{d} g \psi_{1}\left(g h_{1}, \sqrt{j(j+1)}\right) \psi_{3}\left(g h_{2}, \sqrt{j(j+1)}\right)=\delta\left(h_{1} h_{2}^{-1}\right) .
\end{aligned}
$$

Using the above theorem, we know that

$$
\psi(g, \sqrt{j(j+1)})=\sum_{n m}(\tilde{\psi}(\sqrt{j(j+1)}))_{j}^{n m} D_{n m}^{j}(g),
$$

which results in the condition

$$
\left(\tilde{\psi}_{1}\right)_{j}^{n m_{1}}\left(\tilde{\psi}_{3}\right)_{j}^{n m_{3}}=\delta^{m_{1} m_{3}} \quad \forall j \in \mathbb{N},
$$

which characterizes functions satisfying condition (2).

Using the same distribution $\alpha(\vec{B})$, we can define a function satisfying condition (3) too. Let $f$ be a function of the type 19; we define the distribution $F(g, B)=\alpha(\vec{B}) f(g, B)$ and impose condition (3) to find

$$
\int \mathrm{d} g \int \mathrm{d} \vec{B} F^{2}(g, B)=\int \mathrm{d} g \int \mathrm{d} \vec{B} \alpha^{2}(\vec{B}) f^{2}(g, B)=\sum_{j=0}^{\infty}(2 j+1)^{2} \int \mathrm{d} g f^{2}(g, \sqrt{j(j+1)}) .
$$

If this sum is convergent then we can normalize $f$ so that the result is 1 and condition (3) is satisfied. 
Therefore, using functions satisfying the above conditions, we have found an exact solution of equation (15):

$$
\begin{aligned}
\phi_{123} & =\int \mathrm{d} h \varphi_{1}\left(g_{1} h, B_{1}\right) F\left(g_{2} h, B_{2}\right) \varphi\left(g_{3} h, B_{3}\right) \\
& =\int \mathrm{d} h \alpha\left(\vec{B}_{1}\right) \psi_{1}\left(g_{1} h, B_{1}\right) \alpha\left(\vec{B}_{2}\right) f\left(g_{2} h, B_{2}\right) \alpha\left(\vec{B}_{3}\right) \psi_{3}\left(g_{3} h, B_{3}\right) .
\end{aligned}
$$

Now we write down the effective action for a generic perturbation around such solution:

$$
\begin{aligned}
S[\epsilon]= & \frac{1}{2} \frac{1}{(2 \pi)^{9}} \int[\mathrm{d} g] \int[\mathrm{d} \vec{B}] \epsilon_{123} \hat{O}_{1} \hat{O}_{2} \hat{O}_{3} \epsilon_{321}-\frac{\lambda}{4 !(2 \pi)^{36}} \int[\mathrm{d} g] \int[\mathrm{d} \vec{B}] \epsilon_{123} \epsilon_{345} \epsilon_{526} \epsilon_{641} \\
& -\frac{1}{(2 \pi)^{9}} \int[\mathrm{d} g] \mathrm{d} h_{1} \mathrm{~d} h_{2} \int[\mathrm{d} \vec{B}] \psi_{1}\left(g_{1} h_{1}, B_{1}\right) f\left(g_{2} h_{1}, B_{2}\right) f\left(g_{4} h_{2}, B_{4}\right) \\
& \times \psi_{3}\left(g_{5} h_{2}, B_{5}\right) \Psi\left(h_{1}^{-1} h_{2}\right) \epsilon_{526} \epsilon_{641}-\frac{1}{2} \frac{1}{(2 \pi)^{9}} \int[\mathrm{d} g] \mathrm{d} h_{1} \mathrm{~d} h_{2} \\
& \times \int[\mathrm{d} \vec{B}] \psi_{1}\left(g_{1} h_{1}, B_{1}\right) \psi_{3}\left(g_{3} h_{1}, B_{3}\right) \psi_{1}\left(g_{5} h_{2}, B_{5}\right) \psi_{3}\left(g_{6} h_{2}, B_{6}\right) \xi\left(h_{1}^{-1} h_{2}\right) \epsilon_{345} \epsilon_{641} \\
& -\sqrt{\frac{\lambda}{3 !(2 \pi)^{45}} \int[\mathrm{d} g] \int[\mathrm{d} \vec{B}] \psi_{1}\left(g_{1} h, B_{1}\right) f\left(g_{2} h, B_{2}\right) \psi_{3}\left(g_{3} h, B_{3}\right) \epsilon_{345} \epsilon_{526} \epsilon_{641},}
\end{aligned}
$$

where we have defined

$$
\begin{aligned}
& \Psi\left(h_{1}^{-1} h_{2}\right) \equiv \int \mathrm{d} g \int \mathrm{d} \vec{B} \psi_{3}\left(g h_{1}, B\right) \psi_{1}\left(g h_{2}, B\right) \\
& \xi\left(h_{1}^{-1} h_{3}\right) \equiv \int \mathrm{d} g \int \mathrm{d} \vec{B} f\left(g h_{1}, B\right) f\left(g h_{3}, B\right) .
\end{aligned}
$$

This is a very general result, holding for any solution and any perturbation. Now we would obtain, starting from 25 , an action with a kinetic term of the type $\int \mathrm{d} x \phi(x) K(x) \phi(x)$, either in group or in Lie algebra variables, imposing some particular form of the perturbation field. This turns out to be very difficult, and we have not been able to identify any simple form of the perturbations that would give such simplification. Roughly speaking, it is due to the presence of the terms $\epsilon_{526} \epsilon_{641}$ and $\epsilon_{345} \epsilon_{641}$, which are not equivalent, because we have not assumed any invariance of the GFT field, nor of the corresponding perturbation, under permutations of its arguments. There is no simple choice of the perturbation field that gives a product of the type $\epsilon(x) \epsilon(x)$, for example, no field with a dependence on the $B$ variables only through the linear combination $a B_{1}+b B_{2}+c B_{3}$ or the analog in the $G$ variables. This is true, unless we assume that the fundamental GFT field as well as the classical solution chosen and the corresponding perturbation field are invariant under permutations of their group and Lie algebra arguments. Imposing this property, however, would complicate the analysis considerably. Leaving aside the issue of perturbations, the problem can be seen as due to the non-separability of the solution chosen, in particular, the functions $f$ and $\Psi$. We cannot exclude that a different solution or a more involved choice of perturbation would lead to a nicer effective action. However, we also note that even if one solves somehow this first problem, another trouble comes from the distribution $\alpha(\vec{B})$, which appears in the effective action for the perturbations with different powers, leading to additional technical complications.

This suggests us to leave aside for the moment the issue of perturbations around exact solutions of the generalized model. We turn our attention, instead, to approximate solutions and their corresponding perturbations. 


\subsection{Approximate solutions and perturbations}

The basic idea here is to look for eigenfunctions of the Laplace-Beltrami operator and, at the same time, good approximations of the delta function on the group. We note that every good approximation of the delta function on the group has to be sharply peaked on the identity. Therefore, the Laplace-Beltrami operator applied to such a function will be close to zero everywhere but in a neighborhood of the identity element. For this reason we study now the behavior of the Laplace-Beltrami operator on the group $S U(2)$ which is isomorphic to the 3 -sphere $S_{3}$, very close to the identity element, which we identify with the north pole. Now we fix a chart in a neighborhood of the identity element, and we use polar coordinates. The Laplace-Beltrami operator becomes

$\square=\frac{1}{\sqrt{g}} \partial_{\mu}\left(\sqrt{g} g^{\mu \nu} \partial_{\nu}\right) \approx \frac{1}{\theta^{2}} \frac{\partial}{\partial \theta}\left(\theta^{2} \frac{\partial}{\partial \theta}\right)+\frac{1}{\theta^{2} \sin \phi} \frac{\partial}{\partial \phi}\left(\sin \phi \frac{\partial}{\partial \phi}\right)+\frac{1}{\theta^{2} \sin ^{2} \phi} \frac{\partial^{2}}{\partial \phi^{2}}$.

If we apply this operator to functions $f$ only dependent on the $\theta$ variable, we can consider only the first term in the previous formula, so we can use the operator

$$
\square_{A} f=\frac{1}{\theta^{2}} \frac{\partial}{\partial \theta}\left(\theta^{2} \frac{\partial f}{\partial \theta}\right)=\frac{1}{\theta} \frac{\partial^{2}}{\partial \theta^{2}}(\theta f)
$$

where the $A$ indicates both the approximation in the operator and that we can apply it just on the function of $\theta$ considerably different from zero only in a small neighborhood of $\theta=0$.

Now we consider the eigenvalue problem for this operator: $\square_{A} f(\theta)=\lambda f(\theta)$. Among the solutions of this equation, one is particularly interesting for our purposes $f(\theta)=\frac{\mathrm{e}^{-\frac{\theta}{a}}}{\theta}$, where $\lambda=\frac{1}{a^{2}}$. Indeed we note that in the limit $a \rightarrow 0^{+}$the function $f(\theta)$ goes to zero everywhere but in $\theta=0$ where it goes to infinity. Therefore, with appropriate normalization, this is a good representation of the Dirac delta function. Thus, we define the function

$$
\delta_{a}(\theta)=\frac{\mathrm{e}^{-\frac{\theta}{a}}}{a^{2} \theta}
$$

that satisfies the following properties:

$$
\square \delta_{a}(\theta) \approx \square_{A} \delta_{a}(\theta)=\frac{1}{a^{2}} \delta_{a}(\theta) \quad \lim _{a \rightarrow 0^{+}} \delta_{a}(\theta)=\delta(\theta) .
$$

Of course we can rewrite the $\delta_{a}$ function in terms of the group variable, using the parametrization: $g=\cos \theta \mathbb{I}+\mathrm{i} \sin \theta \hat{n} \cdot \vec{\sigma}$, with $\operatorname{Tr}(g)=2 \cos (\theta)$ and $\hat{n} \in S_{2}$. Through this relation, we can interpret the $\delta_{a}(\theta)$ as a $\delta_{a}(g)$ on $S U(2)$. Now we apply the operator $\hat{O}$ on $\delta_{a}(g)$, with $a \approx 0^{+}$, to find

$$
\left(\square+B^{2}-\frac{m^{2}}{8}\right) \delta_{a}(g) \approx\left(\frac{1}{a^{2}}+B^{2}-\frac{m^{2}}{8}\right) \delta_{a}(g) \approx \frac{1}{a^{2}} \delta_{a}(g) .
$$

Of course the approximation improves as $a$ tends to zero. Indeed we have supposed that $\frac{1}{a^{2}} \gg\left|B^{2}-\frac{m^{2}}{8}\right|$. In order for our solution of the equations of motion to satisfy this condition for all relevant values of $B$, we then assume that it is a function with compact support (i.e. identically zero outside of some bounded set) with respect to the $B$ variables.

Another important point is that we can handle $\delta_{a}(g)$ like a true $\delta(g)$ function, as it satisfies $\int \mathrm{d} g \delta_{a}\left(g h^{-1}\right) f(g) \approx f(h)$. The last step is to introduce another function $\Delta_{a}(\theta)$ on the sphere $S_{3}$. It is defined, for $0 \leqslant \theta<\pi / 2$ (i.e. for the the half-sphere corresponding to $S O(3)$ ), as

$$
\Delta_{a}(\theta)=\frac{1}{2} \frac{1}{\sqrt{\pi a}} \frac{\mathrm{e}^{-\frac{\theta}{a}}}{\theta}=\frac{1}{2 \sqrt{\pi}} a^{\frac{3}{2}} \delta_{a}(\theta)
$$


and it is extended to the whole $S_{3} \approx S U(2)$ (i.e. to $0 \leqslant \theta<\pi$ ), by requiring $\Delta_{a}(g)=-\Delta_{a}(-g)$, again for $a \approx 0$. This function is almost vanishing everywhere but for $g=\mathbb{I},-\mathbb{I}$ and satisfies

$$
\square \Delta_{a}(\theta) \approx \frac{1}{a^{2}} \Delta_{a}(\theta) \quad \int \mathrm{d} g \Delta_{a}(g)=0 \quad \int \mathrm{d} g \Delta_{a}^{2}(g)=1 .
$$

In the last two properties the integration is over $S U$ (2). If we restricted the integration only over $S O(3)$, the first integral gives $2 \sqrt{\pi} a^{3 / 2}$ and the second one gives $1 / 2$.

Finally using all the above, it is easy to verify that the following function is an approximate solution of (15):

$\phi_{123}=\sqrt{\frac{3 !(2 \pi)^{27}}{\lambda a^{6}}} \int \mathrm{d} h \delta_{a}\left(g_{1} h\right) \psi_{1}\left(B_{1}\right) \Delta_{a}\left(g_{2} h\right) f\left(B_{2}\right) \delta_{a}\left(g_{3} h\right) \psi_{3}\left(B_{3}\right)$,

where $\psi_{1}, f, \psi_{3}$ are functions with compact support, dependent only on the module of $\vec{B}$, and satisfy the conditions

$$
\int \mathrm{d} \vec{B} f^{2}(B)=1 \quad \int \mathrm{d} \vec{B} \psi_{1}(B) \psi_{3}(B)=1 .
$$

Next we study perturbations around this new approximate solutions. The effective action for a generic perturbation field $\epsilon\left(g_{1}, g_{2}, g_{3} ; B_{1}, B_{2}, B_{3}\right)$ is

$$
\begin{aligned}
& S[\epsilon] \propto \frac{1}{2} \int[\mathrm{d} g] \int[\mathrm{d} \vec{B}] \epsilon_{123} \hat{O}_{1} \hat{O}_{2} \hat{O}_{3} \epsilon_{321}-\frac{\lambda}{4 !(2 \pi)^{27}} \int[\mathrm{d} g] \int[\mathrm{d} \vec{B}] \epsilon_{123} \epsilon_{345} \epsilon_{526} \epsilon_{641} \\
&-\frac{1}{a^{6}} \int[\mathrm{d} g] \mathrm{d} h \int[\mathrm{d} \vec{B}] \psi_{1}\left(B_{5}\right) \Delta_{a}\left(g_{2} h\right) f\left(B_{2}\right) \Delta_{a}\left(g_{4} h\right) f\left(B_{4}\right) \psi_{3}\left(B_{1}\right) \\
& \times \epsilon\left(h^{-1}, g_{2}, g_{3} ; B_{1}, B_{2}, B_{3}\right) \epsilon\left(g_{3}, g_{4}, h^{-1} ; B_{3}, B_{4}, B_{5}\right) \\
&-\frac{1}{2} \frac{1}{a^{6}} \int[\mathrm{d} g] \mathrm{d} h_{1} \mathrm{~d} h_{2} \int[\mathrm{d} \vec{B}] \psi_{1}\left(B_{3}\right) \Delta_{a}\left(g_{4} h_{1}\right) \psi_{3}\left(B_{5}\right) \psi_{1}\left(B_{6}\right) \Delta_{a}\left(g_{4} h_{2}\right) \\
& \times \psi_{3}\left(B_{1}\right) \epsilon\left(h_{2}^{-1}, g_{2}, h_{1}^{-1} ; B_{1}, B_{2}, B_{3}\right) \epsilon\left(h_{1}^{-1}, g_{2}, h_{2}^{-1} ; B_{5}, B_{2}, B_{6}\right) \\
&-\sqrt{\frac{\lambda}{3 !(2 \pi)^{27} a^{6}} \int[\mathrm{d} g] \mathrm{d} h \int[\mathrm{d} \vec{B}] \psi_{1}\left(B_{6}\right) \Delta_{a}\left(g_{4} h\right) f\left(B_{4}\right) \psi_{3}\left(B_{1}\right)} \\
& \times \epsilon\left(h^{-1}, g_{2}, g_{3} ; B_{1}, B_{2}, B_{3}\right) \epsilon_{345} \epsilon\left(g_{5}, g_{2}, h^{-1} ; B_{5}, B_{2}, B_{6}\right) .
\end{aligned}
$$

Here there is a first difference with respect to the previous case: due to the approximation, the linear terms, which would cancel exactly when perturbing around exact extrema of the action, do not cancel exactly but are nevertheless suppressed with $a$ and we have not written them for this reason.

In order to get a 'good' effective theory we need again to impose some special conditions on the perturbation field. As in the static-ultralocal theory, we have two sets of variables, group and algebra; therefore, we have more room for maneuvering with respect to previous models. The goal is again to obtain a field theory with a kinetic term written in the usual form $\int \mathrm{d} x \phi(x) K(x) \phi(x)$, and thus with a field depending on a single (group or algebra) argument. Moreover we recall that the group and algebra variables have the interpretation of momentum and configuration variables in the usual matter field theory of non-commutative type.

The first assumption is the separability of the perturbation field

$$
\epsilon\left(g_{1}, g_{2}, g_{3} ; B_{1}, B_{2}, B_{3}\right)=\varphi\left(g_{1}, g_{2}, g_{3}\right) \phi\left(B_{1}, B_{2}, B_{3}\right) .
$$

Next, having again in mind the possible interpretation of the $B$ and $g$ variables as configuration and momentum variables, respectively, we try to reduce the dependence of the 
perturbation field to either of the two sectors, by treating the other sector as non-dynamical, as in the static-ultralocal case.

Case 1: dynamical group sector. We recall that we have fixed the value of $a$ very close to zero. Thus we notice immediately that in action (35) we have two dominant quadratic terms. We restrict our attention to them, i.e. to the free field theory sector. The dominant terms, comparing powers of $1 / a$, are

$$
\begin{aligned}
S[\epsilon]= & -\frac{1}{(2 \pi)^{9}} \frac{1}{a^{6}} \int \mathrm{d} g_{2} \mathrm{~d} g_{3} \mathrm{~d} g_{4} \mathrm{~d} h_{1} \int \mathrm{d} \vec{B}_{1} \ldots \mathrm{d} \vec{B}_{5} \psi_{1}\left(B_{5}\right) \Delta_{a}\left(g_{2} h_{1}\right) f\left(B_{2}\right) \Delta_{a}\left(g_{4} h_{1}\right) \\
& \times f\left(B_{4}\right) \psi_{3}\left(B_{1}\right) \epsilon\left(h_{1}^{-1}, g_{2}, g_{3} ; B_{1}, B_{2}, B_{3}\right) \epsilon\left(g_{3}, g_{4}, h_{1}^{-1} ; B_{3}, B_{4}, B_{5}\right) \\
& -\frac{1}{2} \frac{1}{(2 \pi)^{9}} \frac{1}{a^{6}} \int \mathrm{d} g_{2} \mathrm{~d} g_{4} \mathrm{~d} h_{1} \mathrm{~d} h_{2} \int \mathrm{d} \vec{B}_{1} \mathrm{~d} \vec{B}_{2} \mathrm{~d} \vec{B}_{3} \mathrm{~d} \vec{B}_{5} \mathrm{~d} \vec{B}_{6} \psi_{1}\left(B_{3}\right) \Delta_{a}\left(g_{4} h_{1}\right) \psi_{3}\left(B_{5}\right) \\
& \times \psi_{1}\left(B_{6}\right) \Delta_{a}\left(g_{4} h_{2}\right) \psi_{3}\left(B_{6}\right) \epsilon\left(h_{2}^{-1}, g_{2}, h_{1}^{-1} ; B_{1}, B_{2}, B_{3}\right) \epsilon\left(h_{1}^{-1}, g_{2}, h_{2}^{-1} ; B_{5}, B_{2}, B_{6}\right) .
\end{aligned}
$$

Now we separate the variables like in (36) and we impose the usual condition $\varphi\left(g_{1}, g_{2}, g_{3}\right)=\varphi\left(g_{1} g_{3}^{-1}\right)$. After integrating out $B$ variables, treating the part of the perturbation field depending on them as non-dynamical, we get

$$
S[\varphi]=\frac{1}{2} \frac{1}{(2 \pi)^{9}} \frac{C}{a^{6}} \int \mathrm{d} g \varphi(g) K(g) \varphi\left(g^{-1}\right),
$$

where the kinetic operator is

$$
K(g)=\int \mathrm{d} h \Delta_{a}(h) \Delta_{a}(h g),
$$

and we have defined the constant

$$
C=\int \mathrm{d} \vec{B}_{1} \vec{B}_{2} \vec{B}_{3} \vec{B}_{5} \vec{B}_{6} \psi_{1}\left(B_{3}\right) \psi_{3}\left(B_{5}\right) \psi_{1}\left(B_{6}\right) \psi_{3}\left(B_{1}\right) \phi_{123} \phi_{526}
$$

We see that we obtain once more a nice field theory on a single group manifold, interpreted as (curved) momentum space, whose dynamics is again entirely determined by the classical GFT solution chosen, thus by the quantum spacetime we choose when selecting the solution itself, and on which the effective scalar field propagates. Once more, we could Fourier transform from group to Lie algebra space, playing the role of effective spacetime, introducing the appropriate star product.

Case 2: dynamical Lie algebra sector. Here we restrict to the $S U(2)$ case. Without this restriction our choice of perturbation field gives a non-local effective action. This case, from a technical point of view, is very similar to the static-ultralocal limit. Indeed we choose exactly the same type of perturbation field:

$$
\epsilon_{123}=\frac{1}{\sqrt{V_{B}}} \delta\left(\vec{B}_{1}-\vec{B}_{2}\right) \psi\left(\vec{B}_{2}+\vec{B}_{3}\right),
$$

where $V_{B}$ is again the (truncated) volume of $\mathbb{R}^{3}$. After a straightforward calculation, we get

$$
S[\psi]=\frac{1}{128} \int \mathrm{d} \vec{B} \psi(\vec{B}) K(B) \psi(\vec{B})+\frac{\lambda}{4 !} \int \mathrm{d} \vec{B} \psi^{4}(\vec{B})
$$

where $K(B)=\left(B^{2}-\frac{m^{2}}{2}\right)^{3}$. This is a nice local effective action with a rather unusual kinematical term, with an algebraic dependence on the configuration space Lie algebra variable. Once more, the specific form of the kinetic term could possibly be modified by different choices of classical (approximate) solution or perturbation field, even though it is not straightforward to envisage how to do so. What cannot be modified easily is again the fact that 
we find no sign of the expected underlying non-commutativity in this field theory, and that the conjugate nature of $B$ and $g$ sectors is lost. This confirms that something is dubious in the way the original GFT model, i.e. the generalized GFT formalism, because we can once more trace back the origin of this limitation in the effective field theory to the way the generalized GFT formalism deals with the dependence on the GFT field on the Lie algebra $B$ variables.

\section{Discussion and conclusions}

Let us summarize the results we have obtained. We have studied the issue of classical solutions of the generalized 3D GFT model proposed in [30-32], and of the effective dynamics of the corresponding perturbations, trying to identify specific solutions and perturbations such that the dynamics of the latter can be understood as that of a scalar field on an effective (noncommutative) spacetime.

The motivations were as follows. First of all, to improve our understanding of such models, at a more technical level. Second, to show that indeed effective matter field theories could be extracted as phases of such generalized models. This means on the one hand again solving the technical difficulties coming from the more involved structure of this model, as compared to the easier ones which have been dealt with up to now, but on the other hand showing that the idea of matter as emerging from the collective dynamics of the micro-constituents of quantum spacetime is more solid than could be originally thought, holding for a wider class of GFT models. Third, and conversely, to use this procedure for extracting effective matter theory from GFT as a test for the generalized GFT formalism itself. This means learning from the way this strategy for emergent matter is made to succeed or from the way it fails, in this context, what are the weak and dubious points of the formalism.

Our results are indeed mixed. We have managed to identify exact solutions of the GFT equations of motion both in the simpler static-ultralocal truncation of the model and in the full, more complicated, one. In the truncated model we could extract rather straightforwardly an effective scalar field theory for the perturbations. When only the group sector of the model is treated as dynamical, the effective field theory takes indeed the expected form of a noncommutative field theory written in momentum space. One could then use a non-commutative Fourier transform to rewrite the theory in configuration space, given by the $\mathfrak{s u}(2)$ Lie algebra. Because of the presence of both Lie algebra and group variables in the fundamental GFT model, one could also expect that it should be possible to obtain directly a non-commutative field theory for the perturbations in configuration space, by treating directly the only Lie algebra sector of the perturbations as dynamical. This turns out not to be the case. One obtains indeed an effective scalar field theory on the same Lie algebra, but without the wanted and expected non-commutative structure. This turn out to be the result both in the truncated model and in the full model, for which we have been able to extract a nice(r) effective dynamics for perturbations, i.e. one that could be interpreted as a scalar field theory on an emergent 3D spacetime, only within a certain approximation, that is only around approximate solutions of the equations of motion. Moreover, a closer look at the way the solutions of the equations of motions could be found and the effective dynamics for perturbations extracted shows that it has been necessary, also when dealing with the full model, to basically trivialize the kinetic term of the theory to the level of the corresponding equations, i.e. look for solutions for which the non-trivial part of the kinetic term gives a basically trivial contribution to the equations. While this last point, although somehow disappointing, could be argued to be solvable with more work and some more clever analysis of the same equations, the first point appears to us to be more fundamental, i.e. to suggest a fundamental limitation of the generalized GFT formalism itself. Moreover, we have worked with special types of classical solutions of the 
GFT equations, separable in the three pairs of group+algebra variables. Again, we cannot exclude that some of the difficulties we encountered in deriving satisfactory effective noncommutative field theories for matter are due to this restriction. While this is true, however, it also true that, again, the first point, i.e. the lack of non-commutative structure in the $B$ variables, seems still more fundamental than this somewhat practical limitation.

Let us be more precise. The generalized GFT formalism of [30-32] is based on the idea of enlarging the set of variables on which the GFT field depends from group elements alone to include Lie algebra elements as well, interpreted as discrete connection and $B$ field of a BF-based description of simplicial geometry, which should become manifest at the level of the Feynman amplitudes. The two sets of variables are treated on equal footing and in parallel, while maintaining the GFT an ordinary, even if non-local with respect to the combinatorics of arguments, field theory. This implies two choices: first, treating $\mathfrak{s u ( 2 )}$ as an ordinary vector space, thus neglecting its non-commutative nature, and second, relaxing the conjugate nature (at the classical level) of $B$ and $g$ variables in the definition of the model (which characterizes the BF theory), and more specifically in the choice of kinetic term. Some consequences of these choices from the point of view of simplicial geometry are discussed in [31, 32]. The results we have presented concerning the effective dynamics of GFT perturbations, in particular the 'negative' ones, suggest that the way the Lie algebra $B$ variables are introduced and treated in the generalized GFT formalism is not correct. The apparent need to trivialize the kinetic term in the choice of classical solution suggests that only when the conjugate nature of the $B$ and $g$ variables is implemented, and thus a BF kinematics is chosen, the corresponding classical solutions define an effective (quantum) flat spacetime as desired. The lack of non-commutative structure in the effective action in the Lie algebra sector suggests instead that the correct way of introducing the $B$ variables in the formalism is not to enlarge the domain space of the GFT field, but to use from the start a non-commutative Fourier transform (in particular, the one introduced and studied in $[13,34,35]$ to go from group space to Lie algebra space, turning the original formulation of GFTs into a non-commutative (and combinatorially non-local) one). All these suggestions are indeed taken into account in the new non-commutative formulation of GFTs developed in [36], which, on top of solving several difficulties we encountered here in the study of perturbations, brings many additional bonuses and further insights from the point of view of simplicial geometry and fundamental quantum gravity proper.

\section{Appendix. The distribution $\alpha(\vec{B})$}

In this appendix, we introduce in a geometrical way the distribution $\alpha(\vec{B})$. The geometric idea is shown in figure 1 where we have suppressed one dimension. Over each corona, we build a cylindrical one such that each one is built around a 2 -sphere of radius $\sqrt{j(j+1)}$ and has thickness $2 b$. Just like the Dirac delta function, we then send $b$ to zero keeping the volume of each corona fixed. Let us define a set of functions

$$
f_{j}(\vec{B} ; b)= \begin{cases}\frac{(2 j+1)^{2}}{V_{b}(j)} & \sqrt{j(j+1)}-b \leqslant|\vec{B}| \leqslant \sqrt{j(j+1)}+b \\ 0 & \text { elsewhere }\end{cases}
$$

where $b$ is a positive, but 'small', number and $V_{b}(j)=\frac{4 \pi}{3}\left[2 b^{3}+6 j(j+1) b\right]$ is the 3 -volume of the $j$ th spherical corona for $j \in \mathbb{N}$ and $j>0$. Moreover, we define $V_{b}(0)=\frac{4 \pi}{3} b^{3}$ and 


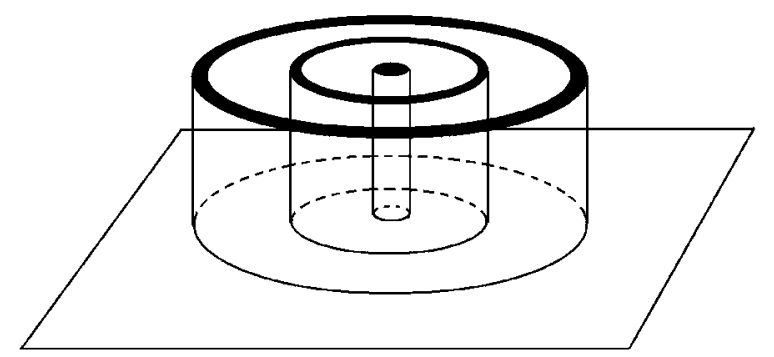

Figure 1. Geometric idea of the distribution $\alpha(\vec{B})$.

$$
f_{0}(\vec{B} ; b)= \begin{cases}\frac{1}{V_{b}(0)} & 0 \leqslant|\vec{B}| \leqslant b \\ 0 & \text { elsewhere. }\end{cases}
$$

Each $f_{j}$ represents a cylindrical corona, while $f_{0}$ is the central cylinder. So we have our distribution

$$
\alpha(\vec{B})=\lim _{b \rightarrow 0^{+}} \sqrt{\sum_{j=0}^{\infty} f_{j}(\vec{B}, b)} .
$$

Of course it is possible to define our distribution through smoother functions, e.g. Gaussianlike functions centered in $|\vec{B}|=\sqrt{j(j+1)}$, and then follow the same procedure shown above, with the same result.

Now, suppose we need to calculate an integral of the type

$$
\int \mathrm{d} \vec{B} \alpha^{2}(\vec{B}) f(|\vec{B}|) \text {. }
$$

From a geometric point of view, the height of the $j$ th cylinder, of volume $(2 j+1)^{2}$, is multiplied by $f(\sqrt{j(j+1)})$. Therefore, the resulting volume is (with $f$ having spherical symmetry):

$$
\int \mathrm{d} \vec{B} \alpha^{2}(\vec{B}) f(B)=\sum_{j=0}^{\infty}(2 j+1)^{2} f(\sqrt{j(j+1)}) .
$$

\section{References}

[1] Oriti D (ed) 2009 Approaches to Quantum Gravity: Toward a New Understanding of Space, Time and Matter (Cambridge: Cambridge University Press)

[2] Oriti D 2009 The group field theory approach to quantum gravity Approaches to Quantum Gravity: Toward a New Understanding of Space, Time and Matter (Cambridge: Cambridge University Press) (arXiv:gr-qc/0607032)

[3] Oriti D 2006 Quantum gravity as a quantum field theory of simplicial geometry Quantum Gravity ed B Fauser, J Tolksdorf and E Zeidler (Basel: Birkhauser) arXiv:gr-qc/0512103

[4] Freidel L 2005 Group field theory: an overview Int. J. Theor. Phys. 441769 (arXiv:hep-th/0505016)

[5] Giddings S B and Strominger A 1989 Baby universe, third quantization and the cosmological constant $N u c l$. Phys. B 321 481-508

[6] Freidel L, Gurau R and Oriti D 2009 Group field theory renormalization-the 3d case. Power counting of divergences Phys. Rev. D 80044007 (arXiv:0905.3772 [hep-th])

[7] Magnen J, Noui K, Rivasseau V and Smerlak M 2009 Scaling behaviour of three-dimensional group field theory Class. Quantum Grav. 26185012 (arXiv:0906.5477 [hep-th])

[8] Gurau R 2009 Colored group field theory arXiv:0907.2582 [hep-th] 
[9] Ben-Geloun J, Magnen J and Rivasseau V 2009 Bosonic colored group field theory arXiv:0911.1719 [hep-th]

[10] Thiemann T 2007 Modern Canonical Quantum General Relativity (Cambridge: Cambridge University Press)

[11] Rovelli C 2006 Quantum Gravity (Cambridge: Cambridge University Press)

[12] Freidel L and Louapre D 2004 Ponzano-Regge model revisited: I. Gauge fixing, observables and interacting spinning particles Class. Quantum Grav. 215685 (arXiv:hep-th/0401076)

[13] Freidel L and Livine E 2006 Ponzano-Regge model revisited: III. Feynman diagrams and effective field theory Class. Quantum Grav. 232021 (arXiv:hep-th/0502106)

[14] Oriti D and Tlas T 2006 Causality and matter propagation in 3-d spin foam quantum gravity Phys. Rev. D 74104021 (arXiv:gr-qc/0608116)

[15] Oriti D and Pfeiffer H 2002 A spin foam model for pure gauge theory coupled to quantum gravity Phys. Rev. D 66124010 (arXiv:gr-qc/0207041)

[16] Speziale S 2007 Coupling gauge theory to spinfoam 3d quantum gravity Class. Quantum Grav. 245139 (arXiv:0706.1534 [gr-qc])

[17] Hamber H 2007 Discrete and continuum quantum gravity arXiv:0704.2895 [hep-th]

[18] Loll R 1998 Discrete approaches to quantum gravity in four dimensions Living Rev. Rel. 113 (arXiv:gr-qc/9805049)

[19] Freidel L, Oriti D and Ryan J 2005 A group field theory for 3-D quantum gravity coupled to a scalar field arXiv:gr-qc/0506067

Oriti D and Ryan J 2006 Group field theory formulation of 3-d quantum gravity coupled to matter fields Class. Quantum Grav. 236543 (arXiv:gr-qc/0602010)

[20] Dowdall R 2009 Wilson loops, geometric operators and fermions in 3d group field theory arXiv:0911.2391 [gr-qc]

[21] Bilson-Thompson S, Markopoulou F and Smolin L 2007 Quantum gravity and the standard model Class. Quantum Grav. 243975 (arXiv:hep-th/0603022)

He S and Wan Y 2008 Conserved quantities and the algebra of Braid excitations in quantum gravity Nucl. Phys. B 804286 (arXiv:0805.0453 [hep-th])

Markopoulou F and Premont-Schwarz I 2008 Conserved topological defects in non-embedded graphs in quantum gravity Class. Quantum Grav. 25205015 (arXiv:0805.3175 [gr-qc])

[22] Kribs D and Markopoulou F 2005 Geometry from quantum particles arXiv:gr-qc/0510052

[23] Fairbairn W J and Livine E R 2007 3d spinfoam quantum gravity: matter as a phase of the group field theory Class. Quantum Grav. 24 5277-97 (arXiv:gr-qc/0702125)

[24] Girelli F, Livine E and Oriti D 2010 4d deformed special relativity from group field theories Phys. Rev. D 81024015 (arXiv:0903.3475 [gr-qc])

[25] Oriti D 2009 Emergent non-commutative matter fields from group field theory models of quantum spacetime J. Phys. Conf. Ser. 174012047 (arXiv:0903.3970 [hep-th])

[26] Szabo R J 2003 Quantum field theory on noncommutative spaces Phys. Rep. 378207

[27] Majid S 1995 Foundations of Quantum Group Theory (Cambridge: Cambridge University Press)

[28] Camelia G A 2002 Quantum gravity phenomenology: status and prospectus Mod. Phys. Lett. A 17899

[29] Camelia G A 2002 Doubly special relativity Nature 418 34-5 (arXiv:gr-qc/0207049)

[30] Oriti D and Tlas T 2008 A new class of group field theories for 1st order discrete quantum gravity Class. Quantum Grav. 25085011

[31] Oriti D 2009 Group field theory and simplicial quantum gravity arXiv:0902.3903 [gr-qc]

[32] Oriti D and Tlas T 2009 Encoding simplicial quantum geometry in group field theories arXiv:0912.1546 [gr-qc]

[33] Livine E 2009 Matrix models as non-commutative field theories on $\mathbb{R}^{3}$ Class. Quantum Grav. 26195014 (arXiv:0811.1462 [gr-qc])

[34] Freidel L and Majid S 2008 Noncommutative harmonic analysis, sampling theory and the Duflo map in $2+1$ quantum gravity Class. Quantum Grav. 25045006 (arXiv:hep-th/0601004)

[35] Joung E, Mourad J and Noui K 2009 Three dimensional quantum geometry and deformed Poincaré symmetry J. Math. Phys. 50052503 (arXiv:0806.4121 [hep-th])

[36] Baratin A and Oriti D 2010 A non-commutative representation for group field theories and simplicial quantum gravity arXiv:1002.4723 [hep-th] 